\title{
Downregulated miRNA-324-5p aggravates neuronal injury induced by oxygen-glucose deprivation via modulating RAN
}

\author{
JUNQUAN GU* , SHUHUA GUI* , LINLIN HU, LIANG KONG, MEIQI DI and YINMING WANG \\ Department of Neurology, The Third People's Hospital of Wuxi, Wuxi, Jiangsu 214041, P.R. China
}

Received September 6, 2019; Accepted November 5, 2019

DOI: $10.3892 /$ etm.2019.8249

\begin{abstract}
Differentially expressed miRNAs in the GEO profile of ischemic stroke were analyzed to clarify the specific role of microRNA-324-5p (miRNA-324-5p) in ischemic stroke and the potential mechanism. After screening out miRNA-324-5p, its level in peripheral blood of stroke patients and in vitro oxygen-glucose deprivation (OGD)-induced primary rat neurons was determined by quantitative real-time polymerase chain reaction (qRT-PCR). Regulatory effects of miRNA-324-5p on viability, and apoptosis of OGD-induced neurons were evaluated by CCK-8 and Annexin V fluorescein isothiocyanate (FITC)/propidium iodide (PI) staining, respectively. Glucose uptake and caspase-3 activity in OGD-induced neurons transfected with miRNA-324-5p mimics or inhibitor were also examined. The binding of miRNA-324-5p to its target gene RAN was analyzed by dual-luciferase reporter gene assay and western blot analysis. By analyzing the data of GSE46266 profile, miRNA-324-5p expression was shown markedly lower in MCAO rats relative to controls. Identically, we also observed the downregulated miRNA-324-5p in peripheral blood of stroke patients and in vitro OGD-induced primary neurons. Overexpression of miRNA-324-5p accelerated viability, induced apoptosis and strengthened glucose uptake ability of OGD-induced neurons. Knockdown of miRNA-324-5p, conversely, obtained the opposite results. Furthermore, we confirmed the binding of miRNA-324-5p to RAN, the target gene that was negatively regulated by miRNA-324-5p. Importantly, RAN overexpression partially reversed the regulatory effect of miRNA-324-5p on viability and glucose uptake of OGD-induced neurons. miRNA-324-5p is downregulated after ischemic stroke, which aggravates the disease condition by inhibiting neuronal proliferation and glucose uptake via upregulating RAN.
\end{abstract}

Correspondence to: Dr Yinming Wang, Department of Neurology, The Third People's Hospital of Wuxi, 585 Xinyuanbei Road, Liangxi, Wuxi, Jiangsu 214041, P.R. China

E-mail: rfrwc072@163.com

${ }^{*}$ Contributed equally

Key words: miRNA-324-5p, oxygen-glucose deprivation, neuronal injury, RAN

\section{Introduction}

Ischemic stroke is a cerebrovascular disease that seriously threatens human health. High rates of incidence, mortality, disability and recurrence, as well as many serious complications are the characteristics of ischemic stroke. With the improved life quality and fast-paced lifestyle, the incidence of ischemic stroke has markedly increased and the disease onset is becoming younger. So far, thrombolysis is the most effective treatment for ischemic stroke $(1,2)$. However, thrombolysis has a strict time window limitation. Only 5\% of ischemic stroke patients are eligible for thrombolysis, and unfortunately, they suffer from thrombolysis-related bleeding risk (3). Therapeutic efficacy of thrombolysis is not satisfactory. Only $50 \%$ ischemic stroke patients are able to achieve recanalization after thrombolysis, and they may also experience revascularization in the future (4). Therefore, it is urgent to search for novel targets for clinical treatment of ischemic stroke.

In recent years, many studies have reported that microRNAs (miRNAs) are able to regulate pathological processes of cerebral ischemia in different stages (5). miRNAs are a class of non-coding small RNAs with 19-23 nt in length that are highly conserved in sequence (6). miRNAs degrade or inhibit translation of mRNAs at post-transcriptional level and are completely or incompletely complementary to them. They participate in various biological progressions, including development, differentiation, innate immune response and adaptive immune response (7). Currently, there are hundreds of miRNAs discovered in human brain, which are involved in the development and pathophysiology of the nervous system (8).

miRNA-324-5p is located on chromosome 17p13.1. Deficiency of miRNA-324-5p in medulloblastoma is proved to be associated with del (17p) (9). In addition, studies have confirmed that miRNA-324-5p regulates metastasis, invasion, stemness and drug-resistance of hepatocellular carcinoma $(10,11)$. The role of miRNA-324-5p in ischemic stroke, however, remains unclear.

\section{Patients and methods}

Sample collection. A total of 80 cases of acute ischemic stroke patients admitted to the Third People's Hospital of Wuxi (Wuxi, China) from July 2015 to March 2017 were enrolled. Moreover, 80 cases of healthy controls undergoing physical examination at the same period were enrolled as controls. Written informed 
consent was obtained before the study, which was approved by the Ethic Committee of the Third People's Hospital of Wuxi.

Analysis of differentially expressed miRNAs. Analysis of differentially expressed miRNAs was performed using the GEO2R online analysis tool (http://www.ncbi.nlm.nih. gov/geo/geo2r/) of the GEO database. GEO2R identifies the differentially expressed genes by variance analysis and t-test using the R project for statistical computing. Genes with a fold difference $\geq 1.5$ and $\mathrm{P}<0.05$ were considered to be differentially expressed.

Primary neuron extraction. After anesthetizing and disinfecting the pregnant rats, gestational sac was harvested and placed in sterile Hank's Balanced Salt Solution (HBSS). The epidermis and skull were cut with ophthalmology, and the cortical brain tissue was harvested using an elbow microscopic sputum. After peeling off meninges and blood vessels, cortical tissues were cut, digested in $0.125 \%$ trypsin at $37^{\circ} \mathrm{C}$ for $15 \mathrm{~min}$ and cultured. Primary neurons were seeded in a 6-well plate and NB27 medium was applied 2 h later. At 7 days of culture, primary neurons were collected for subsequent experiments.

Establishment of oxygen-glucose deprivation (OGD) model in primary neurons. Primary neurons were cultured in glucose-free Dulbecco's modified Eagle's medium (DMEM) (Gibco; Thermo Fisher Scientific, Inc.) and maintained in a three-gas incubator for $2 \mathrm{~h}$. Neurons were then incubated in glucose-containing DMEM and maintained at $37^{\circ} \mathrm{C}, 5 \% \mathrm{CO}_{2}$ incubator for 12, 24 and $48 \mathrm{~h}$, respectively.

Glucose uptake determination. Primary neurons were seeded in the 24-well plate and glucose uptake percentage was determined using the glucose oxidase-peroxidase method. Neurons were stimulated with synthetic insulin $(100 \mathrm{nmol} / \mathrm{l})$ for $6 \mathrm{~h}$. The supernatant of each well was collected, centrifuged at $1,500 \mathrm{x} \mathrm{g}$ at $4^{\circ} \mathrm{C}$ for $5 \mathrm{~min}$ and subjected to absorbance determination. Percentage of glucose uptake was finally calculated.

Caspase-3 activation determination. Primary neurons were lysed in $100 \mu \mathrm{l}$ of lysate solution and the supernatant transferred into the pre-cold Eppendorf (EP) tube, then incubated with $10 \mu$ l of Ac-DEVD-pNA (2 mmol/l) for 60-120 min. After color change, absorbance at $405 \mathrm{~nm}$ was determined.

Cell transfection. Transfection was performed when the confluence was up to $80-90 \%$ following the instructions of Lipofectamine 2000 (Invitrogen; Thermo Fisher Scientific, Inc.). The final dose of miRNA-324-5p mimics and inhibitor was adjusted to $50 \mathrm{nmol} / 1$.

Cell apoptosis determination. Primary neurons were incubated with $10 \mu 1$ of Annexin V fluorescein isothiocyanate (FITC) and $5 \mu \mathrm{l}$ of propidium iodide (PI) in the dark. Finally, cells were suspended in $1 \mathrm{X}$ binding buffer for $20 \mathrm{~min}$ in dark, followed by flow cytometry detection (Partec AG).

Western blot analysis. Total protein was extracted from cell lysis, quantified and electrophoresed. After transferring on polyvinylidene fluoride (PVDF) membranes (EMD Millipore), they were incubated with primary antibodies at $4^{\circ} \mathrm{C}$. The following day, membranes were incubated with the corresponding secondary antibody for $2 \mathrm{~h}$. Bands were exposed with enhanced chemiluminescence, and integral optical density was analyzed by gel imaging analysis system (NIH).

RNA extraction and quantitative real-time polymerase chain reaction ( $q R T-P C R)$. We used TRIzol (Invitrogen; Thermo Fisher Scientific, Inc.) to extract total RNA. Cell lysis was mixed with chloroform, centrifuged at $12,000 \mathrm{x} \mathrm{g}$ at $4^{\circ} \mathrm{C}$ for $10 \mathrm{~min}$ and the precipitate was incubated with isopropanol. After centrifugation, the precipitate was washed with $75 \%$ ethanol, air dried and diluted in diethyl pyrocarbonate (DEPC) water (Beyotime). The extracted RNA was subjected to reverse transcription using the Revert Aid First Strand cDNA Synthesis kit (Thermo Fisher Scientific, Inc.) and amplified by SYBR $^{\circledR}$-Green Master Mix (Takara). Glyceraldehyde 3-phosphate dehydrogenase (GAPDH) was used as the internal reference. Primer sequences were as follows: miRNA-324-5p, forward, GCTATCACAGAGCATTTTCTCAT and reverse, TGCACCAAACACGACTTTTAACC; RAN, forward, GGT GGTACTGGAAAAACGACC and reverse, CCCAAG GTGGCTACATACTTCT.

Dual-luciferase reporter gene assay. RAN 3'-untranslated region (3'UTR) containing the wild-type or mutant-type sequences of the miRNA-324-5p target binding sites was cloned into the luciferase reporter vector, respectively. It was co-transfected with miRNA-324-5p mimics/NC in neurons for $48 \mathrm{~h}$, followed by luciferase activity determination.

Cell counting kit-8 (CCK-8) assay. Primary neurons were seeded in a 96 -well plate with $5 \times 10^{3}$ cells per well. At the appointed time-points, $10 \mu \mathrm{l}$ of CCK-8 (Dojindo) solution was added to each well and incubated at $37^{\circ} \mathrm{C}$ for $2 \mathrm{~h}$. The wavelength at $450 \mathrm{~nm}$ was detected by a microplate reader.

Statistical analysis. Data were analyzed by Statistical Product and Service Solutions (SPSS) 20.0 statistical software (IBM, Corp.). Quantitative data were represented as mean \pm standard deviation (mean $\pm \mathrm{SD}$ ) and analyzed by the t-test. $\mathrm{P}<0.05$ was considered as statistically significant.

\section{Results}

Downregulated miRNA-324-5p in ischemic stroke. We downloaded the miRNA profile GSE46266 from the GEO database and analyzed the differentially expressed miRNAs in ischemic stroke rats (Fig. 1A). It is indicated that miRNA-324-5p was markedly downregulated in MCAO rats relative to controls (Fig. 1B). Peripheral blood samples of ischemic stroke patients and normal samples were collected in the Third People's Hospital of Wuxi. Identically, miRNA-324-5p level remained lower in blood samples of ischemic stroke patients (Fig. 1C). Subsequently, we constructed the in vitro model of ischemic stroke by OGD induction in primary rat neurons. As qRT-PCR data revealed, miRNA-324-5p level was downregulated by OGD induction, and gradually decreased with the prolongation of reperfusion (Fig. 1D). 
A

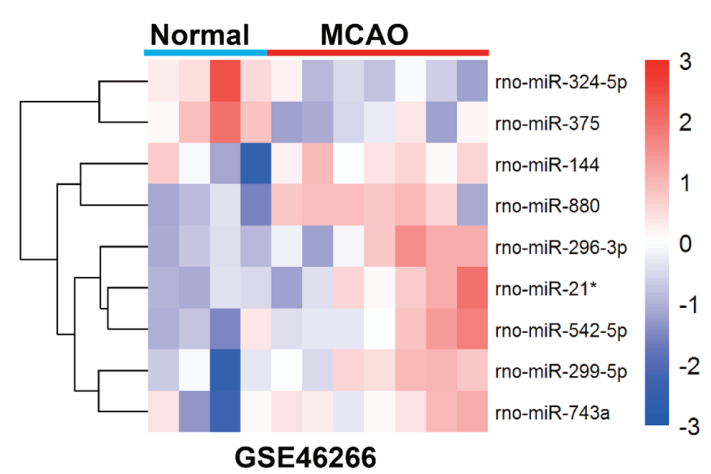

C

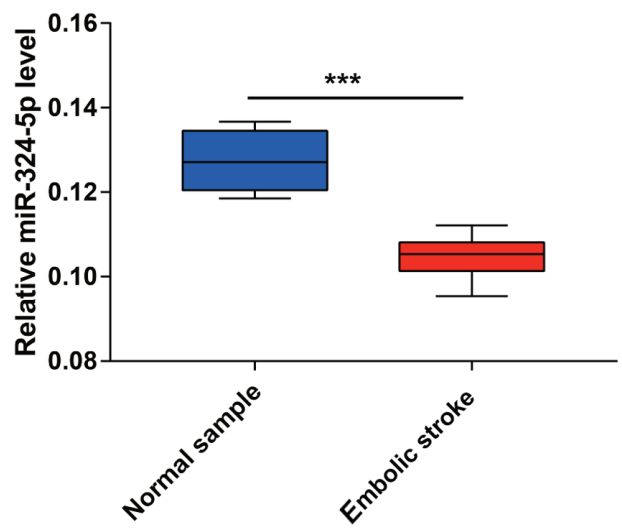

B

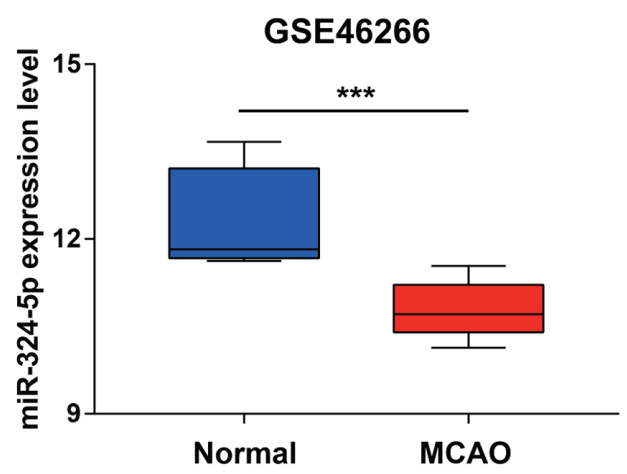

D

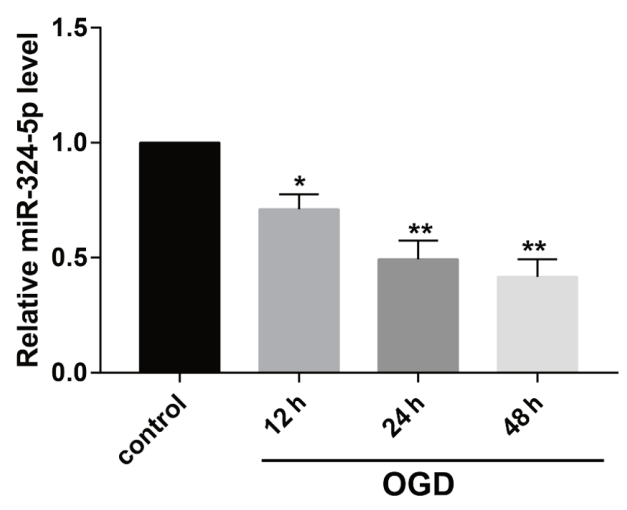

Figure 1. Downregulated miR-324-5p in ischemic stroke. (A) The miRNA profile GSE46266 from the GEO database. (B) GSE46266 dataset showed that miR-324-5p was markedly downregulated in MCAO rats relative to controls (compared with normal, $\left.{ }^{* * *} \mathrm{P}<0.001\right)$. (C) miR-324-5p was downregulated in blood samples of ischemic stroke patients (compared with normal, $\left.{ }^{* * *} \mathrm{P}<0.001\right)$. (D) miR-324-5p level was downregulated by OGD induction in primary neurons, and gradually decreased with the prolongation of reperfusion (compared with control, ${ }^{*} \mathrm{P}<005,{ }^{* *} \mathrm{P}<0.01$ ).

miRNA-324-5p participates in OGD-induced cerebral ischemic injury. To elucidate the biological function of miRNA-324-5p, we first transfected miRNA-324-5p mimics or inhibitor in OGD-induced primary neurons to test their transfection efficacy (Fig. 2A). Viability was remarkably elevated in OGD-induced primary neurons overexpressing miRNA-324-5p. Conversely, knockdown of miRNA-324-5p achieved the opposite trend (Fig. 2B). Glucose uptake was accelerated by miRNA-324-5p overexpression (Fig. 2D). However, we observed inhibited neuronal apoptosis after miRNA-324-5p overexpression as the decreased caspase-3 activity and apoptotic rate revealed (Fig. 2C and E).

miRNA-324-5p inhibits RAN expression. miRNA is capable of inhibiting the transcription and translation of target mRNAs by binding to them. Here, we predicted the binding between miRNA-324-5p and RAN by bioinformatics method (Fig. 3A). Luciferase activity was remarkably reduced in cells co-transfected with RAN-WT and miRNA-324-5p mimics, whereas it did not change in those transfected with RAN-WT, indicating the binding of RAN to miRNA-324-5p (Fig. 3B). Both mRNA and protein levels of RAN were negatively regulated by miRNA-324-5p (Fig. 3C and D).

RAN overexpression accelerates OGD-induced cerebral ischemic injury. Contrary to the expression pattern of miRNA-324-5p, RAN was gradually upregulated by OGD induction at both mRNA and protein levels (Fig. 4A-C).
Transfection of pcDNA-RAN sufficiently upregulated RAN level in OGD-induced primary neurons (Fig. 4D and E). It was found that RAN overexpression decreased viability and glucose uptake, but enhanced apoptotic rate of primary neurons (Fig. 4F-H).

We speculate the involvement of RAN in miRNA-324-5pmediated ischemic stroke. OGD-induced primary neurons were transfected with miRNA-324-5p mimics or miRNA-324-5p mimics + pcDNA-RAN. Increased viability and glucose uptake due to miRNA-324-5p overexpression were partially reversed by RAN overexpression (Fig. $5 \mathrm{~A}$ and $\mathrm{B}$ ). The data demonstrated that miRNA-324-5p alleviated ischemic stroke by downregulating RAN.

\section{Discussion}

Ischemic brain injury involves complex pathological processes. miRNAs, as novel biological hallmarks, have been identified to be crucial in different stages of cerebral ischemic injury (12-15). In vitro and in vivo experiments have demonstrated that overexpression of miRNA-134 exacerbates cell death and apoptosis. miR-134 deficiency in OGD-induced N2A cells and ischemic brain tissues upregulate the protein level of HSPA128. Moreover, miR-134 knockdown could markedly reduce brain infarct size, alleviate nerve cell damage and elevate neurological function score in mice (16). Sun et al (17) reported that miR-124 overexpression remarkably enlarges cerebral infarction area and 
A

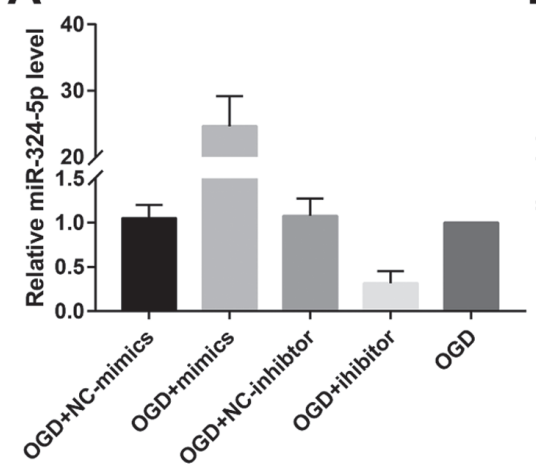

D

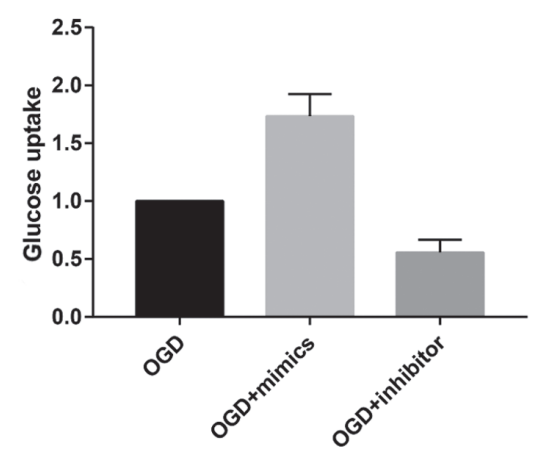

B

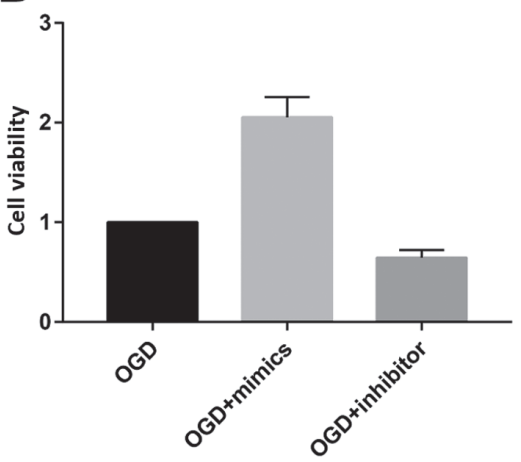

$E$

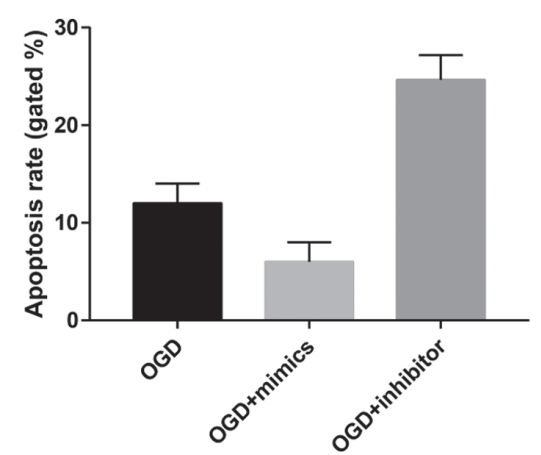

C

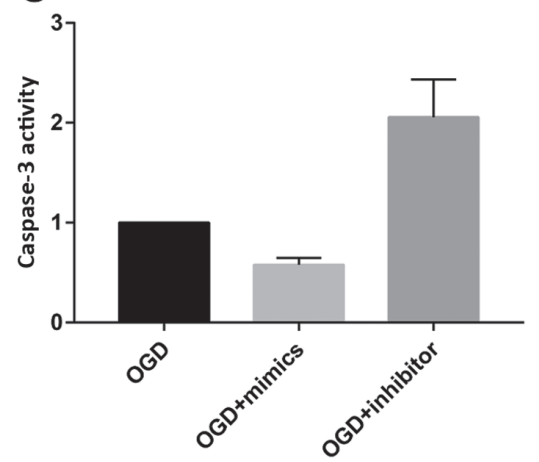

Figure 2. miR-324-5p participates in OGD-induced cerebral ischemic injury. (A) Transfection efficacy of miR-324-5p mimics or inhibitor in OGD-induced primary neurons. (B) Cell viability was remarkably elevated in OGD-induced primary neurons transfected with miR-324-5p mimics, and inhibited by transfection of miR-324-5p inhibitor. (C) Caspase-3 activity was remarkably inhibited in OGD-induced primary neurons transfected with miR-324-5p mimics, and elevated by transfection of miR-324-5p inhibitor. (D) Glucose uptake was remarkably elevated in OGD-induced primary neurons transfected with miR-324-5p mimics, and inhibited by transfection of miR-324-5p inhibitor. (E) Apoptotic rate was remarkably inhibited in OGD-induced primary neurons transfected with miR-324-5p mimics, and elevated by transfection of miR-324-5p inhibitor.

A

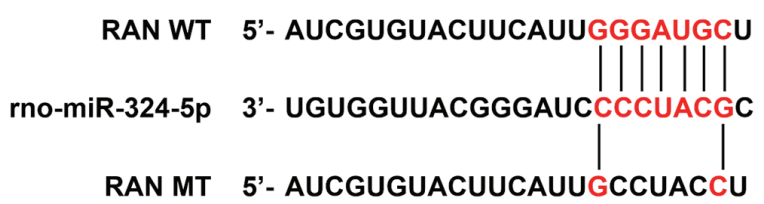

C

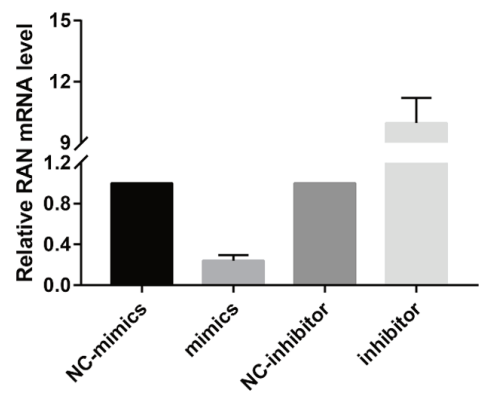

D

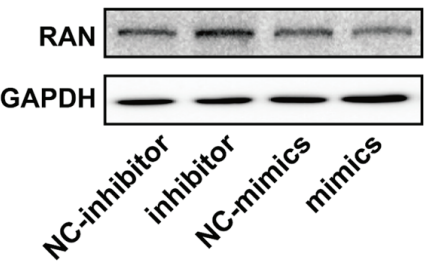

B
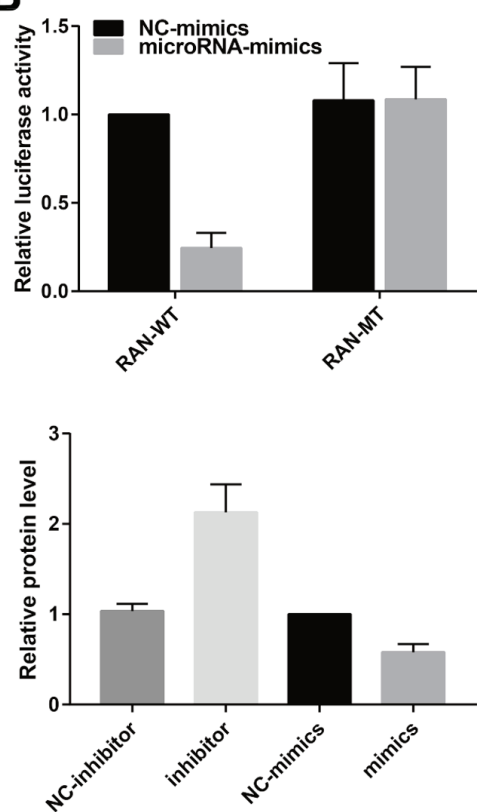

Figure 3. miR-324-5p inhibits RAN expression. (A) Binding sequence between miR-324-5p and RAN predicted by bioinformatics method. (B) Luciferase activity was remarkably reduced in cells co-transfected with RAN-WT and miR-324-5p mimics, whereas it did not change in those transfected with RAN-WT. (C and D) The mRNA (C) and protein levels (D) of RAN were negatively regulated by miR-324-5p.

downregulated miR-124 exerts a protective role in ischemic stroke by inhibiting neuronal apoptosis. Overexpression of
miR-424 alleviates ischemic brain damage by inhibiting G1/S phase transition and microglial activation (18). 
A

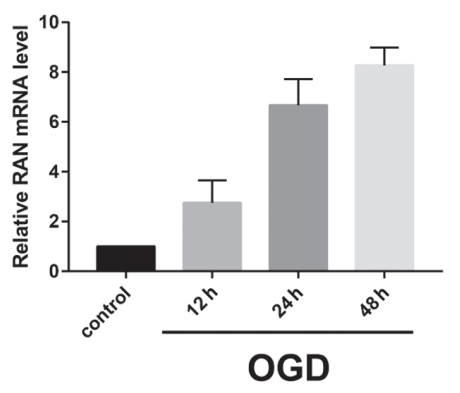

D

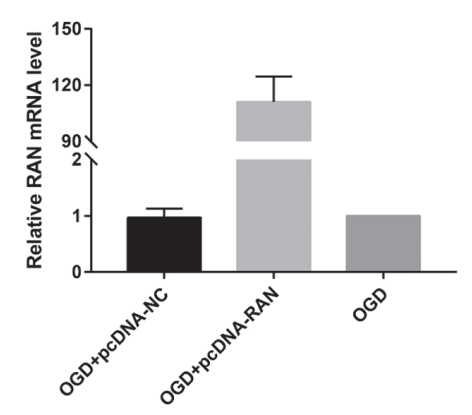

G

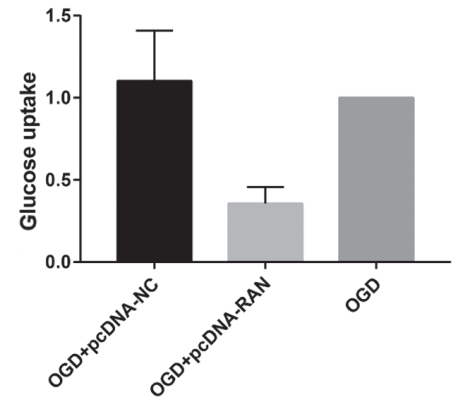

B

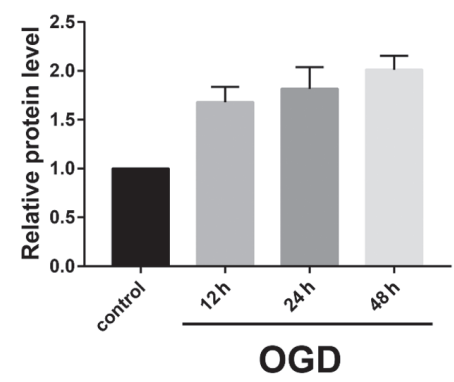

$\mathbf{E}$

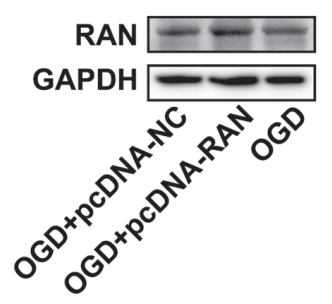

C

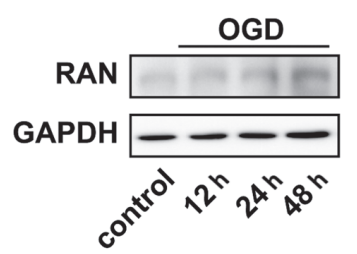

$\mathbf{F}$

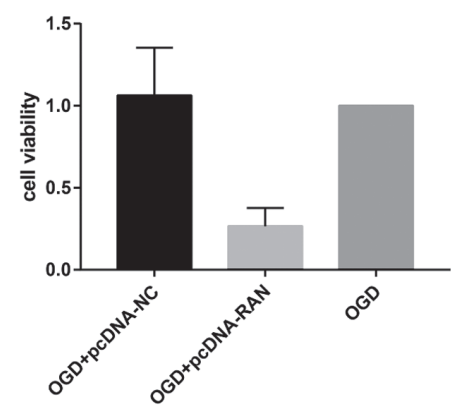

H

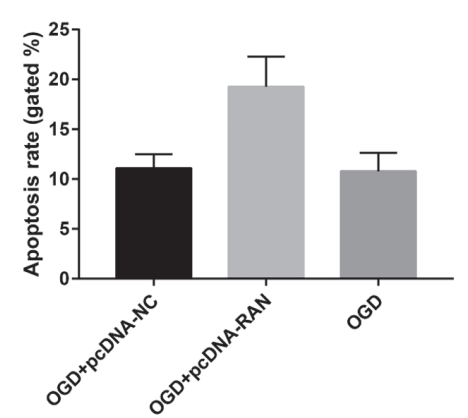

Figure 4. RAN overexpression accelerates OGD-induced cerebral ischemic injury. (A-C) The mRNA (A) and protein levels (B and C) of RAN are gradually upregulated by OGD induction. (D and E) Transfection efficacy of pcDNA-RAN in OGD-induced primary neurons at mRNA (D) and protein levels (E). (F) Cell viability decreased by RAN overexpression. (G) Glucose uptake decreased by RAN overexpression. (H) Apoptotic rate increased by RAN overexpression.
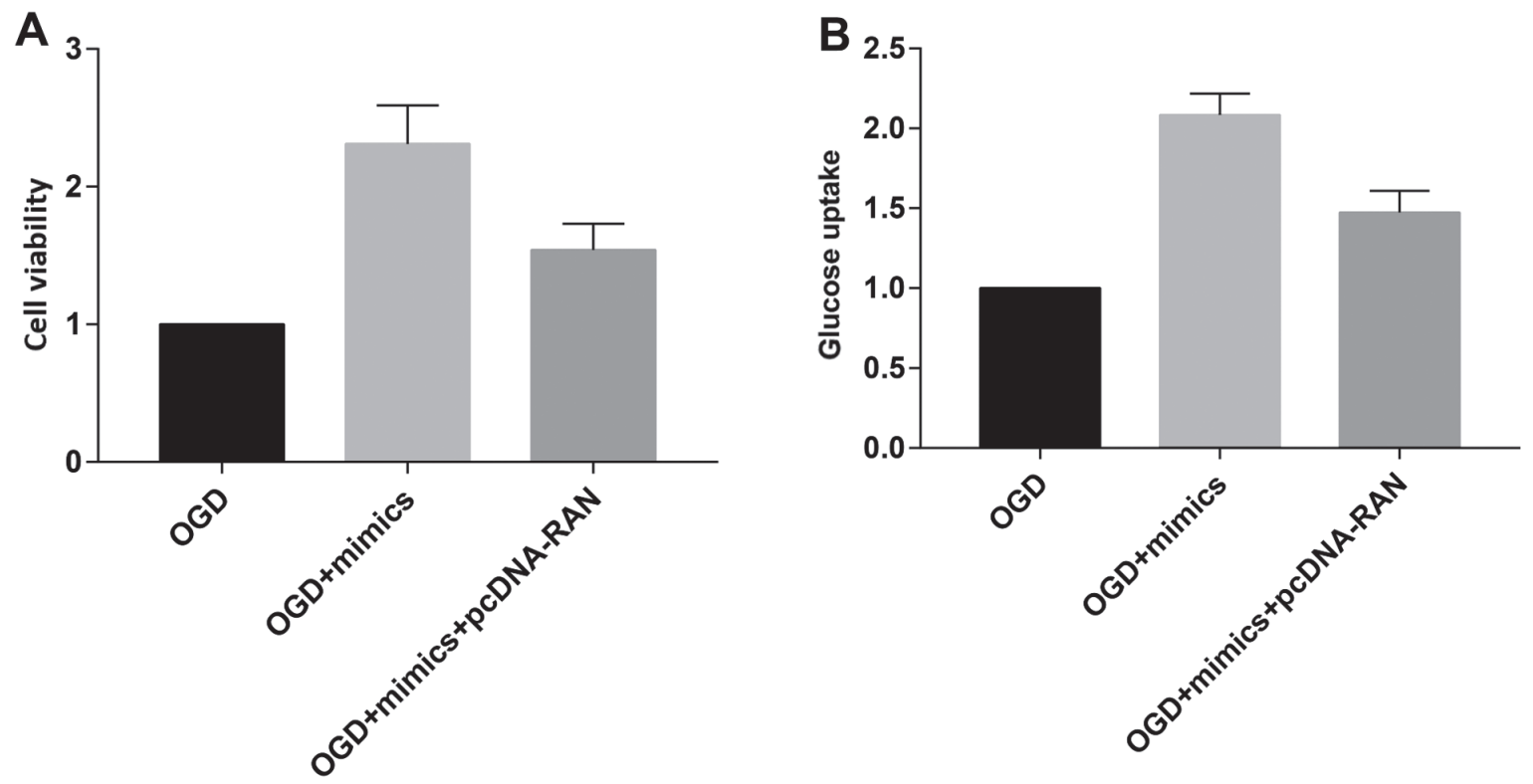

Figure 5. RAN overexpression reverses the role of miR-324-5p. OGD-induced primary neurons were transfected with miR-324-5p mimics or miR-324-5p mimics+pcDNA-RAN. (A) Cell viability in treated neurons. (B) Glucoseuptake in treated neurons. 
A relevant study screened out the target gene of miRNA-181, GRP78, a classic marker of endoplasmic reticulum stress. miRNA-181 is downregulated in the cerebral ischemic penumbra, which accelerates the progression of cerebral injury by inducing neuronal apoptosis through upregulating GRP78 (19). Differentially expressed miRNAs in circulating cerebral ischemia may serve as diagnostic and prognostic hallmarks (20-22).

In this study, we found that miRNA-324-5p was closely related to cerebral ischemic injury. miRNA-324-5p was identified to be downregulated in the selected GEO profile, peripheral blood of stroke patients and OGD-induced primary neurons. Overexpression of miRNA-324-5p accelerated viability, induced apoptosis and strengthened glucose uptake ability of OGD-induced neurons. Subsequently, RAN was predicted to be the target gene of miRNA-324-5p, which was negatively regulated by it.

RAN is a $25 \mathrm{kDa}$ GTPase, distributed in nucleus by binding to GTP or cytoplasm by binding to GDP. RAN exerts biological functions in eukaryotic cells, including nuclear transport, mitosis and formation of nuclear membrane and nuclear pore complexes (23). Recent studies have shown that RAN is associated with cell fates, such as cell death, proliferation, differentiation, immortalization and tumorigenesis. RAN dysfunction will lead to unlimited cell proliferation. By analyzing serous epithelial ovarian cancer, RAN expression was shown negatively correlated to disease prognosis (24). Moreover, transfection of siRNA RAN reduces viability of human-derived tumor cell lines (H1299, DLD-1), suggesting that RAN is an effective antitumor target (25). An in vitro experiment demonstrated that RAN knockdown inhibits HepG2 cells to proliferate, exerting remarkable antitumor function (26). The specific role of RAN in ischemic stroke remains unclear. Our results show that RAN overexpression inhibited viability, glucose uptake and induced apoptosis of OGD-induced primary neurons. Importantly, RAN overexpression partially reversed the regulatory effect of miRNA-324-5p on ischemic stroke.

Due to the limited experimental conditions, we only examined the biological effects of miRNA-324-5p in vitro. Its protective effect on cerebral ischemia-reperfusion injury and post-ischemic adaptation require validation in an in vivo model.

In conclusion, miRNA-324-5p is downregulated at post-stroke, which aggravates the progression of stroke by inhibiting neuronal proliferation and glucose uptake via upregulating RAN.

\section{Acknowledgements}

Not applicable.

\section{Funding}

No funding was received.

\section{Availability of data and materials}

All data generated or analyzed during this study are included in this published article.

\section{Authors' contributions}

SG, JG and YW designed the study and performed the experiments, SG, LH and LK collected the data, JG and MD analyzed the data, SG, JG and YW prepared the manuscript. All authors read and approved the final manuscript.

\section{Ethics approval and consent to participate}

This study was approved by the Ethics Committee of the Third People's Hospital of Wuxi (Wuxi, China). Signed informed consents were obtained from the patients or the guardians.

\section{Patient consent for publication}

Not applicable.

\section{Competing interests}

The authors declare no competing interests.

\section{References}

1. Joliat GR, Halkic N, Pantet O and Ben-Hamouda N: Ischemic stroke and ST-elevation myocardial infarction revealing infective endocarditis. Eur Rev Med Pharmacol Sci 21: 4640-4641, 2017.

2. Zhang B, Sun XJ and Ju CH: Thrombolysis with alteplase 4.5-6 hours after acute ischemic stroke. Eur Neurol 65: 170-174, 2011.

3. Koroshetz WJ: Tissue plasminogen activator for acute ischemic stroke. N Engl J Med 334: 1405-1406, 1996.

4. Andonova S, Kirov F and Bachvarov C: The impact of recanalization on ischemic stroke outcome: A clinical case presentation. Perspect Med 1: 455-458, 2012.

5. Ouyang YB and Giffard RG: MicroRNAs regulate the chaperone network in cerebral ischemia. Transl Stroke Res 4: 693-703, 2013.

6. Guo H, Ingolia NT, Weissman JS and Bartel DP: Mammalian microRNAs predominantly act to decrease target mRNA levels. Nature 466: 835-840, 2010.

7. Cardoso AL, Guedes JR, Pereira de Almeida L and Pedroso de Lima MC: miR-155 modulates microglia-mediated immune response by down-regulating SOCS-1 and promoting cytokine and nitric oxide production. Immunology 135: 73-88, 2012.

8. Berezikov E, Thuemmler F, van Laake LW, Kondova I, Bontrop R, Cuppen E and Plasterk RH: Diversity of microRNAs in human and chimpanzee brain. Nat Genet 38: 1375-1377, 2006.

9. Mukhopadhyay D and Riezman H: Proteasome-independent functions of ubiquitin in endocytosis and signaling. Science 315: 201-205, 2007.

10. Cao L, Xie B, Yang X, Liang H, Jiang X, Zhang D, Xue P, Chen D and Shao Z: miR-324-5p suppresses hepatocellular carcinoma cell invasion by counteracting ECM degradation through post-transcriptionally downregulating ETS1 and SP1. PLoS One 10: e0133074, 2015.

11. Xu HS, Zong HL, Shang M, Ming X, Zhao JP, Ma C and Cao L: MiR-324-5p inhibits proliferation of glioma by target regulation of GLI1. Eur Rev Med Pharmacol Sci 18: 828-832, 2014.

12. Zhao H, Tao Z, Wang R, Liu P, Yan F, Li J, Zhang C, Ji X and Luo Y: MicroRNA-23a-3p attenuates oxidative stress injury in a mouse model of focal cerebral ischemia-reperfusion. Brain Res 1592: 65-72, 2014

13. Li LJ, Huang Q, Zhang N, Wang GB and Liu YH: miR-376b-5p regulates angiogenesis in cerebral ischemia. Mol Med Rep 10: 527-535, 2014.

14. Liu XS, Chopp M, Zhang RL, Tao T, Wang XL, Kassis H, Hozeska-Solgot A, Zhang L, Chen C and Zhang ZG: MicroRNA profiling in subventricular zone after stroke: MiR-124a regulates proliferation of neural progenitor cells through Notch signaling pathway. PLoS One 6: e23461, 2011.

15. Buller B, Liu X, Wang X, Zhang RL, Zhang L, Hozeska-Solgot A, Chopp $\mathrm{M}$ and Zhang ZG: MicroRNA-21 protects neurons from ischemic death. FEBS J 277: 4299-4307, 2010. 
16. Chi W, Meng F, Li Y, Wang Q, Wang G, Han S, Wang P and Li J: Downregulation of miRNA-134 protects neural cells against ischemic injury in N2A cells and mouse brain with ischemic stroke by targeting HSPA12B. Neuroscience 277: 111-122, 2014.

17. Sun Y, Gui H, Li Q, Luo ZM, Zheng MJ, Duan JL and Liu X: MicroRNA-124 protects neurons against apoptosis in cerebral ischemic stroke. CNS Neurosci Ther 19: 813-819, 2013.

18. Zhao H, Wang J, Gao L, Wang R, Liu X, Gao Z, Tao Z, Xu C, Song J, Ji X, et al: MiRNA-424 protects against permanent focal cerebral ischemia injury in mice involving suppressing microglia activation. Stroke 44: 1706-1713, 2013.

19. Ouyang YB, Lu Y, Yue S, Xu LJ, Xiong XX, White RE, Sun X and Giffard RG: miR-181 regulates GRP78 and influences outcome from cerebral ischemia in vitro and in vivo. Neurobiol Dis 45: 555-563, 2012.

20. Li P, Teng F, Gao F, Zhang M, Wu J and Zhang C: Identification of circulating microRNAs as potential biomarkers for detecting acute ischemic stroke. Cell Mol Neurobiol 35: 433-447, 2015.

21. Long G, Wang F, Li H, Yin Z, Sandip C, Lou Y, Wang Y, Chen C and Wang DW: Circulating miR-30a, miR-126 and let-7b as biomarker for ischemic stroke in humans. BMC Neurol 13: 178 , 2013.

22. Sepramaniam S, Tan JR, Tan KS, DeSilva DA, Tavintharan S, Woon FP, Wang CW, Yong FL, Karolina DS, Kaur P, et al: Circulating microRNAs as biomarkers of acute stroke. Int J Mol Sci 15: 1418-1432, 2014.
23. Nagai M and Yoneda Y: Small GTPase Ran and Ran-binding proteins. Biomol Concepts 3: 307-318, 2012.

24. Ouellet V, Le Page C, Guyot MC, Lussier C, Tonin PN, Provencher DM and Mes-Masson AM: SET complex in serous epithelial ovarian cancer. Int J Cancer 119: 2119-2126, 2006.

25. Morgan-Lappe SE, Tucker LA, Huang X, Zhang Q, Sarthy AV, Zakula D, Vernetti L, Schurdak M, Wang J and Fesik SW: Identification of Ras-related nuclear protein, targeting protein for xenopus kinesin-like protein 2 , and stearoyl-CoA desaturase 1 as promising cancer targets from an RNAi-based screen. Cancer Res 67: 4390-4398, 2007.

26. Li L, Wang R, Wilcox D, Zhao X, Song J, Lin X, Kohlbrenner WM, Fesik SW and Shen Y: Tumor vasculature is a key determinant for the efficiency of nanoparticle-mediated siRNA delivery. Gene Ther 19: 775-780, 2012

This work is licensed under a Creative Commons Attribution-NonCommercial-NoDerivatives 4.0 International (CC BY-NC-ND 4.0) License. 